\begin{tabular}{|c|l|}
\hline Title & $\begin{array}{l}\text { Impact of the Characteristic Impedance of Coaxial Lines on the Sensitivity of a 750-MHz Electronically Tunable EPR } \\
\text { Resonator }\end{array}$ \\
\hline Author(s) & Nakaoka, Ririko; Komarov, Denis A.; Matsumoto, Shingo; Hirata, Hiroshi \\
\hline Citation & $\begin{array}{l}\text { A pplied magnetic resonance, 49(8), 853-867 } \\
\text { https://doi.org/10.1007/300723-018_1001-6 }\end{array}$ \\
\hline Issue Date & 2018-08 \\
\hline Doc URL & http://hdl.handle.net/2115/75089 \\
\hline Rights & $\begin{array}{l}\text { Thisis a post-peer-review, pre-copyedit version of an article published in A pplied Magnetic Resonance. The final } \\
\text { authenticated version is available online at: https://doi.org/10.1007/s00723-018-1001-6. }\end{array}$ \\
\hline Type & article (author version) \\
\hline File Information & APMR-D-18-00019R1_Manuscript_body.pdf \\
\hline
\end{tabular}

Instructions for use 
Special Issue: SEST-15, Applied Magnetic Resonance

Manuscript No. APMR-D-18-00019R1

\section{Impact of the characteristic impedance of coaxial lines on the sensitivity of a 750-MHz electronically tunable EPR resonator}

Ririko Nakaoka, Denis A. Komarov, Shingo Matsumoto, Hiroshi Hirata*

Division of Bioengineering and Bioinformatics, Graduate School of Information Science and Technology, Hokkaido University, North 14, West 9, Kita-ku, Sapporo, 060-0814, Japan

* Corresponding Author

Hiroshi Hirata, Ph.D.

Division of Bioengineering and Bioinformatics

Graduate School of Information Science and Technology

Hokkaido University

North 14, West 9, Kita-ku

Sapporo, 060-0814

Japan

Phone \& Fax: +81-11-706-6762

E-mail: hhirata@ist.hokudai.ac.jp 


\begin{abstract}
A 750-MHz electronically tunable resonator was investigated in terms of the sensitivity of electron paramagnetic resonance (EPR) signal detection. The conversion efficiency of the RF magnetic field was calculated for resonators with 50- and 100-ohm coaxial coupling lines using three-dimensional (3D) microwave field and microwave circuit simulators. Based on the simulation results, two tunable resonators were physically constructed and compared in terms of EPR signal sensitivity using a nitroxyl radical solution. While the resonator with 100-ohm coaxial lines provided 14\% greater signal intensity, its signal-to-noise ratio was lower than that of the resonator with 50-ohm lines. To demonstrate the capability of the constructed tunable resonator for EPR imaging experiments, a solution of nitroxyl radical and the leg of a tumor-bearing mouse were visualized.
\end{abstract}




\section{Introduction}

Electron paramagnetic resonance (EPR) imaging is a molecular imaging modality that can be used to measure paramagnetic species. With appropriate spin probes, it can also be used to measure oxygen partial pressure, $\mathrm{pH}$, and redox status in small animals [1-3]. For in vivo EPR imaging at a radio-frequency (RF), the high sensitivity of EPR detection is always desirable, because the energy gap in the Zeeman splitting is smaller than that at a higher magnetic field. The RF resonator used in a continuous-wave (CW) EPR spectrometer is a key component to achieve highly sensitive EPR signal detection. While pulsed EPR spectrometers require RF resonators with a low quality factor to reduce the ring-down time [4], an RF resonator with a high quality factor and a high filling factor is needed for a higher sensitivity in CW-EPR spectroscopy and imaging. This is because the EPR signal intensity is proportional to the quality factor, $Q$, and the filling factor, $\eta$ [5]. In the general expression for EPR signal detection, the signal voltage at the resonator, $V_{S}$, is given as

$$
V_{S}=\chi^{\prime \prime} \eta Q \sqrt{P Z_{0}}
$$

where $\chi^{\prime \prime}$ is the imaginary component of the RF magnetic susceptibility, $P$ is the RF power applied to the resonator, and $Z_{0}$ is the characteristic impedance of the transmission line [6]. Note that the signal voltage $V_{S}$ also depends on the characteristic impedance of the transmission line and the applied RF power.

In the linear response region, the EPR signal intensity is proportional to the RF magnetic field, $B_{1}$ [7]. Therefore, the conversion efficiency of the RF magnetic field of an EPR resonator is an important parameter that reflects the sensitivity of EPR detection. Recently, a 750-MHz electronically tunable resonator was developed for use with a tumor-bearing mouse leg [8]. In that resonator design, a multi-coil parallel-gap resonator (MCPGR) [9] is connected to the matching and tuning network with a parallel transmission line formed by two coaxial lines. Commercially available 50-ohm coaxial lines are commonly 
used in EPR spectrometers. However, coaxial lines with a higher characteristic impedance could potentially increase the EPR signal voltage. Although surface coil resonators with 70-ohm transmission lines have been reported [10,11], the impact of the characteristic impedance of coaxial lines on the recently developed tunable resonator remains unclear.

The purpose of this study was to clarify the impact of the characteristic impedance of the coaxial lines used in the 750-MHz tunable resonator on the sensitivity of EPR detection. To understand the behavior of the RF characteristics of the resonator, a three-dimensional (3D) finite-element model of the MCPGR and a model of its feeding circuit were constructed as in the previous report [8]. Based on the simulation results, two tunable resonators with 50and 100-ohm coaxial lines were physically constructed and compared in terms of EPR signal intensity and susceptibility to noise. In the present work, we examined how the characteristic impedance of the coaxial lines used in the tunable resonator affects the conversion efficiency of the RF magnetic field and the sensitivity of EPR detection. Finally, the constructed tunable resonator was used for in vivo 3D EPR imaging of a tumor-bearing mouse leg.

\section{Methods}

\subsection{Resonator specifications}

There were several requirements for the tunable resonator used in CW-EPR spectroscopy and imaging in the present study. The central frequency of the tunable resonator was adjusted to $750 \mathrm{MHz}$ for both the numerical simulations and the physically constructed resonators, because the constant magnetic field of the permanent magnet of the EPR spectrometer was $27 \mathrm{mT}$. For EPR imaging of tumor-bearing mouse legs, the sample space in the MCPGR was chosen to be $16 \mathrm{~mm}$ in diameter and $20 \mathrm{~mm}$ in length. To use the tunable resonator with an automatic tuning control system that controls the resonant frequency of the resonator to the carrier frequency of the CW-EPR spectrometer, the tunable resonator should 
have an appropriate tuning frequency band [12]. In the previous design, the tunable frequency band of the resonator was $4 \mathrm{MHz}$ [8]. However, based on our experience, a tunable frequency band of $2 \mathrm{MHz}$ is enough to compensate the perturbation due to the motion of a subject

mouse during data acquisition. In addition, we found that reducing the tunable frequency band increases the conversion efficiency of the RF magnetic field and could potentially improve the sensitivity of EPR detection. The tunable frequency band was adjusted by choosing the coupling capacitors connected in series between the coaxial lines and the MCPGR.

\subsection{Structure and modeling of an electronically tunable resonator}

The details of the 750-MHz tunable resonator using microstrip line couplers and varactor diodes were previously reported [8]. Figure 1 shows the circuit diagram of the electronically tunable resonator using microstrip line couplers. In brief, the MCPGR was formed by three conductive loops fixed on a sleeve made of cross-linked polystyrene (REXOLITE 1422, C-Lec Plastics Inc., Philadelphia, PA). The capacitors $C_{p}$ and $C_{s}$ (Fig. 1) were made of a copper-clad dielectric substrate (0.38 mm in thickness, CuFlon, 15-7-7, Polyflon Company, Norwalk, CT). The MCPGR was placed in the RF shield made of REXOLITE and covered with strips of copper foil (50 $\mu \mathrm{m}$ in thickness and $10 \mathrm{~mm}$ in width). The resonator assembly was connected to the tuning and matching circuit through a parallel transmission line of 3/4 wavelengths formed with two non-magnetic semi-rigid coaxial lines (5.0 mm in diameter and $207 \mathrm{~mm}$ in length, SC-500/50 for 50 ohms, SC-500/100 for 100 ohms, COAX Corporation, Yokohama, Japan). The diameter of the dielectric insulator (polytetrafluoroethylene, PTFE) in the coaxial lines was $4.18 \mathrm{~mm}$, and the diameters of the center conductor were $1.27 \mathrm{~mm}$ for the 50 -ohm coaxial line and $0.37 \mathrm{~mm}$ for the $100-\mathrm{ohm}$ coaxial line. Attenuation losses of the coaxial lines were $0.28 \mathrm{~dB} / \mathrm{m}$ for the 50 -ohm coaxial line (SC-500/50) and $0.35 \mathrm{~dB} / \mathrm{m}$ for the $100-$ ohm coaxial line (SC-500/100) at $1 \mathrm{GHz}$ (as 
reported by COAX Corporation). The other end of the parallel transmission line was connected to the microstrip line couplers used for frequency tuning. The microstrip line couplers are discussed in the previous report [8]. In brief, the characteristic impedance of the microstrip line was $50 \Omega$ and the measured insertion loss and coupling of the microstrip line couplers were $0.36 \mathrm{~dB}$ and $12.2 \mathrm{~dB}$, respectively, at $750 \mathrm{MHz}$. The microstrip lines were fed through the half-wave line balun made of a 50-ohm non-magnetic semi-rigid coaxial line (2.2 mm in diameter, 138 mm in length, SC-219/50-SC, COAX Corporation, Yokohama, Japan). The attenuation loss of the coaxial line used for the balun was $0.64 \mathrm{~dB} / \mathrm{m}$ at $1 \mathrm{GHz}$.

The MCPGR and RF shield were placed at the center of a Helmholtz coil pair on 1.6-mm-thick glass-reinforced epoxy laminated plates (FR4 equivalent). To increase the mechanical robustness, the plates of the Helmholtz coil pair were glued to 2-mm-thick glass-reinforced epoxy plates. The capacitors $C_{p}$ (see Fig. 1) were made of the copper-clad dielectric substrate, $10 \mathrm{~mm} \times 22 \mathrm{~mm} \times 0.38 \mathrm{~mm}$, by partially removing copper foil from one side. The capacitance $C_{p}$ was $4.3 \mathrm{pF}$ and the coupling capacitance $C_{s}$ was $1.5 \mathrm{pF}$ for the resonator with 50-ohm coaxial lines. For the resonator with 100 -ohm coaxial lines, the dimensions of the copper-clad dielectric substrate were $10 \mathrm{~mm} \times 28 \mathrm{~mm} \times 0.38 \mathrm{~mm}$ and the capacitance $C_{p}$ was $5.5 \mathrm{pF}$ when the coupling capacitance $C_{s}$ was $1.0 \mathrm{pF}$. These values were determined from the simulation results regarding the RF characteristics described below. The REXOLITE sleeves of the MCPGR and RF shield were glued to a 1-mm-thick Bakelite plate, which was attached to the modulation coils.

The characteristics of the tunable resonator were simulated using an HFSS 3D full-wave microwave field simulator and Electronics Desktop 2017.2 by ANSYS Inc. (Canonsburg, PA). Figure 2a shows a 3D finite-element model of the MCPGR including the conductive loops, the parallel capacitors, $C_{p}$, and the coupling capacitors, $C_{s}$. For the simplicity of presentation, the RF shield is not shown, although it was included in the model. The space of $120 \mathrm{~mm} \times$ 
$120 \mathrm{~mm} \times 120 \mathrm{~mm}$ was modeled with a radiation boundary condition. The MCPGR was placed in the center of the simulated space. Figure $2 \mathrm{~b}$ illustrates the cross-sectional view of the structure of the MCPGR, the RF shield, and the REXOLITE sleeves. The parallel transmission line and the half-wave line balun were simulated using a model of coaxial lines from Electronics Desktop. The parameters of the microstrip line couplers were the same as reported previously [8]. The entire model of the tunable resonator built in Electronics Desktop is shown in Fig. 2c. Varactor diodes (variable capacitors) were used for frequency tuning (BB181, NXP Semiconductors, Netherlands) and impedance matching (1SV217, Toshiba Semiconductor, Japan) of the resonator. These varactor diodes were modeled by lumped capacitors that exhibit no loss. The values of these lumped capacitors were manually controlled within the range of the corresponding varactor diodes in the circuit simulator.

\subsection{Simulation of RF characteristics}

To calculate the conversion efficiency of the RF magnetic field, the coupling capacitance $C_{s}$ was varied in the range of 0.5 to $4.0 \mathrm{pF}$. In the computation of the characteristics of the resonator at each coupling capacitance $C_{s}$, the lumped capacitor $C_{m}$ that mimicked the varactor diodes for impedance matching were manually adjusted to achieve critical coupling of the resonator. On the other hand, the lumped capacitors $C_{t}$ that substituted the varactor diodes for frequency tuning were kept constant at $12 \mathrm{pF}$. Note that a change in the coupling capacitance $C_{s}$ also shifts the resonant frequency of the resonator. To keep the resonant frequency in the range of $749-751 \mathrm{MHz}$, we adjusted the capacitance $C_{p}$ by changing the dimensions of the dielectric substrate. In contrast, the tunable frequency band of the resonator was calculated as the difference in the resonant frequencies at the capacitance $C_{t}$ that mimicked the varactor diodes of 2-12 pF. To calculate the conversion efficiency of the RF magnetic field, the generated RF magnetic field at the center of the MCPGR was divided 
by the square root of the input RF power $P$.

\subsection{Measurements of RF characteristics}

The scattering-matrix parameter $S_{11}$ of the tunable resonator was measured with a vector network analyzer (E5062A, Keysight Technologies, Santa Rosa, CA). The RF magnetic field at the center of the MCPGR was measured by a metal sphere perturbing method [13]. To determine the tunable frequency band, a shift in the resonant frequencies at the reverse bias voltage of the tuning control varactor diodes $V_{T}=2 \mathrm{~V}$ and $V_{T}=14 \mathrm{~V}$ was measured. By definition, the unloaded quality (Q) factor refers to the Q-factor for the resonator that is not coupled to a transmission line.

\subsection{EPR spectroscopy}

To investigate the sensitivity of EPR signal detection, we installed the developed tunable resonators into the magnet of a laboratory-built 750-MHz CW-EPR spectrometer/imager. The details of the CW-EPR spectrometer/imager have been reported previously $[14,15]$. A micro-centrifuge tube (9 mm inner diameter and $37 \mathrm{~mm}$ length) that contained $2.0 \mathrm{~mL}$ of a $2 \mathrm{mM}$ solution of nitroxyl radical,

4-oxo-2,2,6,6-tetramethylpiperidine- $\mathrm{d}_{16}, 1-{ }^{15} \mathrm{~N}-1$-oxyl $\left({ }^{15} \mathrm{~N}-\mathrm{PDT}\right.$, CDN Isotopes Inc., Quebec, Canada), in phosphate-buffered saline was placed into the MCPGR. The parameters of EPR spectroscopy were as follows: duration of field scanning $100 \mathrm{~ms}$, magnetic field scanning 5.0 mT, magnetic field modulation $70 \mu \mathrm{T}$, modulation frequency $90 \mathrm{kHz}$, time-constant of a lock-in amplifier $30 \mu$ s, number of data points per scan 2048, number of scans 100 , and incident RF power $5.5 \mathrm{~mW}$. This RF power was below the saturation level of the EPR signals. Although the intrinsic peak-to-peak line-width for the $2 \mathrm{mM}$ solution of ${ }^{15} \mathrm{~N}$-PDT under air-saturated conditions is $60 \mu \mathrm{T}$, the EPR spectrum was recorded under conditions of slight 
over-modulation to obtain a better signal-to-noise ratio (SNR). The SNR was calculated as the peak-to-peak intensity of the first-derivative EPR absorption spectrum divided by the double root mean square (RMS) intensity of the noise signal in the spectral baseline.

\subsection{EPR imaging}

Three-dimensional EPR imaging of the $2 \mathrm{mM}{ }^{15} \mathrm{~N}-\mathrm{PDT}$ radical solution in the micro-centrifuge tube was performed by a CW-EPR-based single-point imaging (SPI) protocol [16-18]. EPR projections were acquired under incrementally ramped field gradients with $15 \times 15 \times 15$ field gradients for the X-, Y-, and Z-directions (total of 3375 projections). The maximum field gradient in each direction was $30 \mathrm{mT} / \mathrm{m}$. Two sets of EPR projections were accumulated to improve the SNR of the resultant EPR image. The total image-acquisition time was $15 \mathrm{~min}$. The central magnetic field was set to the low-field absorption peak of the ${ }^{15} \mathrm{~N}-\mathrm{PDT}$ radical. The other measurement parameters were similar to those in EPR spectroscopy (see Methods 2.5) except for magnetic field scanning $1.5 \mathrm{mT}$, the time-constant of the lock-in amplifier $100 \mu$ s, and the number of data points per scan 512. SPI reconstruction was performed using a delay time of 214 ns in the time domain.

For in vivo EPR imaging of a tumor-bearing mouse leg, a nitroxide radical, trans-3,4-dicarboxy-2,2,5,5-tetra $\left({ }^{2} \mathrm{H}_{3}\right)$ methylpyrrolidin- $\left(3,4-{ }^{2} \mathrm{H}_{2}\right)-\left(1-{ }^{15} \mathrm{~N}\right)-1$-oxyl $\left({ }^{2} \mathrm{H},{ }^{15} \mathrm{~N}-\mathrm{DCP}\right)$, was used as the imaging probe [19]. The ${ }^{2} \mathrm{H},{ }^{15} \mathrm{~N}$-DCP radical was synthesized and kindly provided by Dr. Igor A. Kirilyuk, Novosibirsk Institute of Organic Chemistry, Novosibirsk, Russia. The animal experiments were conducted similar to a procedure that has been reported previously [20]. Murine squamous cell carcinoma (SCC VII) cells were subcutaneously implanted into the right hind legs of seven-week-old C3H HeJ male mice [21]. After a week, when the tumor volume became larger than $0.5 \mathrm{~cm}^{3}$, the tumor-bearing leg of an anesthetized mouse was placed in the MCPGR. The imaging probe ${ }^{2} \mathrm{H},{ }^{15} \mathrm{~N}-\mathrm{DCP}(150 \mu \mathrm{L}$ of a 
$100 \mathrm{mM}$ solution) was intravenously injected as a bolus into the subject mouse through a tail vein (this dose corresponds to $0.75 \mathrm{mmol} / \mathrm{kg}$ body weight for a 20 -g mouse). This dose is comparable to that in previous reports on EPR imaging of mice using nitroxyl radical probes [22,23]. EPR image-acquisition was started 10 minutes after the injection. The EPR imaging parameters were as follows: duration of field scanning $100 \mathrm{~ms}$, magnetic field scanning 1.5 mT, magnetic field modulation $40 \mu \mathrm{T}$, modulation frequency $90 \mathrm{kHz}$, time-constant of the lock-in amplifier $100 \mu \mathrm{s}$, number of data points per scan 512, and incident RF power $2.8 \mathrm{~mW}$. The central magnetic field was set to the low-field absorption peak of ${ }^{2} \mathrm{H},{ }^{15} \mathrm{~N}$-DCP. EPR projections were acquired with the same field gradient scheme as described above. The total image-acquisition time was $7.5 \mathrm{~min}$. SPI reconstruction was performed using a delay time of $167 \mathrm{~ns}$ in the time domain. All animal experiments were performed in accordance with the 'Law for the Care and Welfare of Animals in Japan’ and were approved by the Animal Experiment Committee of Hokkaido University (approval no. 15-0120).

\section{Results and Discussion}

\subsection{Simulated RF characteristics}

To visualize the RF magnetic field inside the MCPGR, the tunable resonator was excited at the resonant frequency in the numerical model. Figure 3a shows the absolute value of the RF magnetic field vector in cross-sectional planes of the MCPGR. The RF magnetic field was calculated for the tunable resonator with 50-ohm coaxial lines, coupling capacitance $C_{s}=1.5 \mathrm{pF}$, and applied RF power of $1 \mathrm{~W}$. At the center of the MCPGR, the RF magnetic field was $48 \mathrm{~A} / \mathrm{m}$, which corresponds to $61 \mu \mathrm{T}$. Some fringe fields were observed at the top and bottom openings of the MCPGR. The magnetic fields were high in close proximity to the conductive loops. However, the central region of the MCPGR had a rather homogeneous distribution of the RF magnetic field, with a deviation of $\pm 10 \%$ for the volume of a 
cylinder-like shape (12 mm in the X-direction, $11 \mathrm{~mm}$ in the Z-direction, and $17 \mathrm{~mm}$ in the Y-direction). The conversion efficiency of the RF magnetic field was estimated to be $61 \mu \mathrm{T} / \mathrm{W}^{1 / 2}$. For the tunable resonator with 100 -ohm coaxial lines and $C_{s}=1.0 \mathrm{pF}$, the calculated conversion efficiency was $75 \mu \mathrm{T} / \mathrm{W}^{1 / 2}$.

Figures 3b and 3c show the conversion efficiency of the RF magnetic field at the center of the MCPGR and the tunable frequency band as a function of coupling capacitance $C_{s}$. While the maximum conversion efficiency was observed at $C_{s}=1.0 \mathrm{pF}$, the tunable frequency band at this coupling capacitance was rather small. Except for the region around $C_{s}=1.0 \mathrm{pF}$, the conversion efficiency of the tunable resonator with 100-ohm coaxial lines was higher than that of the resonator with 50-ohm coaxial lines. Since the EPR signal intensity depends on the conversion efficiency of the RF magnetic field, these results suggest that the resonator with 100-ohm coaxial lines should show better sensitivity for EPR signal detection. Moreover, the tunable frequency band of the resonator with 100-ohm coaxial lines was broader than that of the resonator using 50-ohm coaxial lines. To obtain a tunable frequency band of more than 2 $\mathrm{MHz}$, the coupling capacitance $C_{s}$ should be larger than 1.5 or $1.0 \mathrm{pF}$ for resonators with 50and 100-ohm coaxial lines, respectively.

There are two conditions for impedance matching between the coaxial lines and the microstrip lines in the resonator. Since the microstrip lines had a characteristic impedance of $50 \Omega$, the 50-ohm coaxial lines matched the impedance of the microstrip lines. However, the 100-ohm coaxial lines did not match the characteristic impedance of the microstrip lines. The parallel transmission line formed with two coaxial lines and the microstrip lines are part of the resonator system. Therefore, the difference in impedance matching between the coaxial lines and the microstrip lines affects the distribution of the stored magnetic energy in those transmission lines in the resonator system. The energy distribution is an important consideration for improving resonator sensitivity [24]. The stored energies in the coaxial lines 
and the microstrip lines should be investigated in terms of the optimal design of the resonator.

\subsection{Measured RF characteristics}

Based on the simulation results, the tunable resonators using 50- and 100-ohm coaxial lines were physically constructed. Figure 4a shows photographs of the tunable resonator with 50-ohm coaxial lines. The impedance-matching characteristics (scattering-matrix parameter $S_{11}$ ) are shown in Figure 4b. At a tuning control voltage of $V_{T}=8 \mathrm{~V}$, critical coupling of the empty resonator was achieved when the matching control voltage $V_{M}$ was 7.7 V. Figure 4c shows the dependence of the resonant frequency for resonators with 50- and 100-ohm coaxial lines on the reverse bias voltage applied to the tuning control varactor diodes. At each tuning control voltage, the matching voltage was adjusted to achieve critical coupling of the tunable resonator. For the resonator with 50-ohm coaxial lines, the resonant frequency was shifted from 746.9 MHz to 750.0 MHz (tunable frequency band 3.1 MHz) for the tuning voltage range $V_{T}=2-14 \mathrm{~V}$. The tunable frequency band of the resonator with 100-ohm coaxial lines was $2.1 \mathrm{MHz}$ (from 746.6 to $748.7 \mathrm{MHz}$ ). The measured frequency bands for the constructed resonators were different from what was expected from the simulation results (Fig. 3c). This can be explained by the differences in the capacitance of the varactor diodes, coupling of the microstrip line couplers, and the coupling capacitance between the numerical model and the constructed resonator as well as by stray capacitance in the constructed tunable resonator.

The resonator with 50-ohm coaxial lines had a conversion efficiency of the RF magnetic field of $80 \mu \mathrm{T} / \mathrm{W}^{1 / 2}$ and an unloaded Q-factor of 130. In contrast, the resonator with 100-ohm coaxial lines had a conversion efficiency of $97 \mu \mathrm{T} / \mathrm{W}^{1 / 2}$ and an unloaded Q-factor of 150 . The measured conversion efficiencies were 30\% higher than those expected from the results of the simulation of the RF magnetic field, however, the difference in the conversion efficiencies between resonators with 50- and 100-ohm coaxial lines reasonably agreed with the 
simulations. Note that the tunable resonators built in this study have 2.4- to 2.9-fold higher conversion efficiency than the previously reported resonator, which had the same diameter of the MCPGR and a conversion efficiency of $34 \mu \mathrm{T} / \mathrm{W}^{1 / 2}$ [8]. This result was achieved mainly due to reconsideration of the target tunable frequency band.

\subsection{Signal intensity and SNR}

EPR spectra were recorded with the newly developed tunable resonators with 50-ohm and 100-ohm coaxial lines. Figure 5 shows EPR spectra of $2 \mathrm{mM}{ }^{15} \mathrm{~N}$-PDT radical solution in a $2 \mathrm{~mL}$ micro-centrifuge tube measured using the tunable resonators with 100-ohm coaxial lines (top trace) and 50-ohm coaxial lines (bottom trace). The absolute intensity of the EPR signal was $14 \%$ higher for the tunable resonator with 100 -ohm coaxial lines, which is in good agreement with the results of the simulation of the RF magnetic field. However, the intensity of baseline noise was also higher in the top trace (Fig. 5, insets) and gave SNRs for the EPR spectra of 149 and 134 for resonators with 50- and 100-ohm coaxial lines, respectively.

The SNRs were reproduced for three consecutive measurements, suggesting that, although the tunable resonator with 100-ohm coaxial lines has better EPR sensitivity, its SNR was slightly below that of the resonator with 50-ohm coaxial lines. This result might be related to the higher thermal noise of the resistance in 100 -ohm coaxial lines compared to that in 50-ohm coaxial lines. The increase in noise in the tunable resonator with 100-ohm coaxial lines reduces the SNR despite the higher EPR signal intensity. In light of the present results, we used the tunable resonator with 50-ohm coaxial lines for the following EPR imaging experiments.

The voltage reflection coefficient between the transmission line and the MCPGR decreases with the use of 100-ohm coaxial lines in the resonator. This is because the MCPGR is high-impedance on resonance. This improvement in the reflection coefficient helps to 
transfer the RF magnetic energy to the MCPGR and increase the conversion efficiency of the RF magnetic field. Moreover, eq. (1) suggests that the resonator connected to a high-impedance transmission line gives a higher signal voltage. Since 100-ohm coaxial lines connected to the MCPGR through the coupling capacitors were part of the resonator system, the impedance of coaxial lines does not directly correspond to the impedance of the transmission line in eq. (1). However, it does contribute to an increase in the signal voltage, as shown in Fig. 5.

The sensitivity of EPR detection is related to the signal intensity and the intensity of baseline noise. The RF magnetic field, i.e., the conversion efficiency of the resonator, is directly related to the signal intensity. However, our simulation model of the resonator did not take into account noise in the resonator. An increase in the intensity of baseline noise may be due to the thermal noise in the coaxial lines, because a major difference in the two constructed resonators was the impedance of the coaxial lines of 3/4 wavelengths. Moreover, the reverse bias voltage applied to the varactor diodes used for impedance matching was different for each resonator. This might affect the noise in the varactor diodes. To fully understand the behavior of the sensitivity of the resonator and the SNR of measured EPR signals, noise generated in the resonator should be well formulated and compared to the measured results. A noise analysis is a complex and challenging task that could be conducted in the future to find the optimal design of the resonator.

\subsection{EPR imaging of a solution sample}

To clarify the sensitive region of the constructed tunable resonator, a ${ }^{15} \mathrm{~N}-\mathrm{PDT}$ radical solution was visualized. Figure 6a shows a photograph of a micro-centrifuge tube containing $2 \mathrm{~mL}$ of $2 \mathrm{mM}{ }^{15} \mathrm{~N}$-PDT solution. The size of the micro-centrifuge tube was $37 \mathrm{~mm}$, which means that the bottom part of the tube was outside of the MCPGR (20 mm long). Figures $6 \mathrm{~b}$ 
and 6c show surface-rendered images of the EPR signal calculated with a threshold of 25\% of the maximum signal intensity and slice-selective maps of the EPR signal intensity in three different planes. The visualized part of the ${ }^{15} \mathrm{~N}-\mathrm{PDT}$ solution in the tube was approximately $24 \mathrm{~mm}$ long, which is greater than the length of MCPGR and can be explained by taking fringe RF magnetic fields into account. The distribution of the RF magnetic field for the MCPGR (Fig. 3a) supports this extension of the sensitive region.

\subsection{In vivo EPR imaging}

To test the stability and sensitivity of the constructed tunable resonator, a tumor-bearing

mouse hind leg was scanned. For the in vivo experiments, the ${ }^{2} \mathrm{H},{ }^{15} \mathrm{~N}$-DCP radical was used as an imaging probe because it has narrow EPR absorption peaks and an in vivo half-life of 25 min [20]. Figure 7a illustrates the placement of the tumor-bearing hind leg inside the MCPGR. With the mouse leg inside, the Q-factor of the tunable resonator was 100, which corresponds to an $\mathrm{RF}$ magnetic field of $62 \mu \mathrm{T} / \mathrm{W}^{1 / 2}$. The resonant frequency of the tunable resonator was also shifted from 750.8 MHz down to 747.2 MHz (-3.6 MHz). Figures 7b and 7c show surface-rendered images of the EPR signal in the tumor-bearing mouse leg calculated with a threshold of $25 \%$ of the maximum signal intensity and slice-selective EPR intensity maps, generated from 3D image data. During EPR data-collection, the tuning control circuit of the tunable resonator evenly compensated for any physiological movements of the subject mouse, which makes it suitable for routine measurements of the tumor-bearing mouse leg.

\section{Conclusions}

In this study, the conversion efficiency of the RF magnetic field of the tunable resonator was improved 2.4- to 2.9-fold compared to that of a previous resonator [8]. We showed that the characteristic impedance of the coaxial lines used in the tunable resonator affects the 
sensitivity of EPR signal detection as well as the noise level in the spectral baseline. The tunable resonator with 50 -ohm coaxial lines provided $10 \%$ better SNR than that with 100-ohm coaxial lines, suggesting that the use of low-impedance coaxial lines may improve the SNR of EPR signals. Further studies will be needed to identify the optimal design of a tunable resonator for in vivo EPR imaging.

\section{Acknowledgements}

The nitroxyl radical probe ${ }^{2} \mathrm{H},{ }^{15} \mathrm{~N}$-DCP used in this study was kindly provided by Dr. Igor A. Kirilyuk, Novosibirsk Institute of Organic Chemistry, Novosibirsk, Russia. The authors appreciate Kumiko Yamamoto and Prof. Osamu Inanami at the Graduate School of Veterinary Medicine, Hokkaido University, for their help in the experiments with tumor-bearing mice. This work was supported in part by JSPS KAKENHI Grant Numbers 26249057 and $16 \mathrm{~K} 12862$. 


\section{References}

1. Matsumoto, S., Hyodo, F., Subramanian, S., Devasahayam, N., Munasinghe, J., Hyodo, E., Gadisetti, C., Cook, J. A., Mitchell, J. B., Krishna, M. C.: J. Clin. Invest. 118, 1965$1973(2008)$

2. Koda, S., Goodwin, J., Khramtsov, V. V., Fujii, H., Hirata, H.: Anal. Chem. 84, 3833$3837(2012)$

3. Fujii, H., Sato-Akaba, H., Emoto, M., Itoh, K., Ishihara Y., Hirata, H.: Magn. Reson. Imaging 31, 130-138 (2013)

4. Sundramoorthy, S. V., Epel, B., Halpern, H. J.: J. Magn. Reson. 240, 45-51 (2014)

5. Eaton, G. R., Eaton, S. S., Barr, D. P., Weber, R. T.: Quantitative EPR, pp. 79-90, Springer-Verlag, Wien (2010)

6. Rinard, G. A., Eaton, S. S., Eaton, G. R., Poole, Jr., C. P., Farach, H. A. in: Poole, Jr., C. P., Farach, H. A. (eds.) Handbook of Electron Spin Resonance, vol. 2, pp. 1-23, Springer-Verlag, New York (1999)

7. Eaton, G. R., Eaton, S. S., Barr, D. P., Weber, R. T.: Quantitative EPR, pp. 37-61, Springer-Verlag, Wien (2010)

8. Amida, T., Nakaoka, R., Komarov, D. A., Yamamoto, K., Inanami, O., Matsumoto, S., Hirata, H.: IEEE Trans. Biomed. Eng. doi: 10.1109/TBME.2017.2743232 (2017)

9. Kawada, Y., Hirata, H., Fujii, H.: J. Magn. Reson. 184, 29-38 (2007)

10. Haga, T., Hirata, H., Lesniwski, P., Rychert, K. M., Williams, B. B., Flood, A. B., Swartz, H. M.: Concepts Magn. Reson. B 43B, 32-40 (2013)

11. Sugawara, H., Hirata, H., Petryakov, S., Lesniewski, P., Williams, B. B., Flood, A. B., Swartz, H. M.: IEEE Trans. Biomed. Eng. 61, 1894-1901 (2014)

12. Hirata, H., Watanabe, H., Kumada, M., Itoh, K., Fujii, H.: NMR Biomed. 17, 295-302 (2004) 
13. Freed, J. H., Leniart, D. S., Hyde, J. S.: J. Chem. Phys. 47, 2762-2773 (1967)

14. Sato-Akaba, H., Fujii, H., Hirata, H.: Rev. Sci. Instrum. 79, 123701 (2008)

15. Sato-Akaba, H., Kuwahara, Y., Fujii, H., Hirata, H.: Anal. Chem. 81, 7501-7506 (2009)

16. Subramanian, S., Devasahayam, N., Murugesan, R., Yamada, K., Cook, J., Taube, A., Mitchell, J. B., Lohman, J. A. B., Krishna, M. C.: Magn. Reson. Med. 48, 370-379 (2002)

17. Matsumoto, K., Chandrika, B., Lohman, J. A. B., Mitchell, J. B., Krishna, M. C., Subramanian, S.: Magn. Reson. Med. 50, 865-874 (2003)

18. Jang, H., Subramanian, S., Devasahayam, N., Saito, K., Matsumoto, S., Krishna, M. C., McMillan, A. B.: Magn. Reson. Med. 70, 1173-1181 (2013)

19. Gorodetsky, A. A., Kirilyuk, I. A., Khramtsov, V. V., Komarov, D. A.: Magn. Reson. Med. 76, 350-358 (2016)

20. Kubota, H., Komarov, D. A., Yasui, H., Matsumoto, S., Inanami, O., Kirilyuk, I. A., Khramtsov, V. V., Hirata, H.: Magn. Reson. Mater. Phy. 30, 291-298 (2017)

21. Suit, H.D., Suchato, C.: Radiology 89, 713-719 (1967)

22. Hyodo, F., Matsumoto, S., Devasahayam, N., Dharmaraj, C., Subramanian, S., Mitchell, J. B., Krishna, M. C.: J. Magn. Reson. 197, 181-185 (2009)

23. Emoto, M. C., Sato-Akaba, H., Hirata, H., Fujii H. G.: Free Radic. Biol. Med. 74, 222$228(2014)$

24. Hirata, H., Walczak, T., Swartz, H. M.: Rev. Sci. Instrum. 72, 2839-2841 (2001) 


\section{Figure Legends}

Fig. 1. Schematic diagram of the 750-MHz electronically tunable resonator using a multi-coil parallel-gap resonator (MCPGR), microstrip line couplers, and varactor diodes for tuning and matching control. The characteristic impedance $Z_{c}$ of the coaxial lines used for the parallel transmission line was 50 or $100 \Omega$ for testing the sensitivity of the tunable resonator.

Fig. 2. Numerical model of the tunable resonator. a Three-dimensional (3D) model of the MCPGR with three conductive loops, $\mathbf{b}$ cross-sectional view of the MCPGR and the RF shield made by strips of copper foil (the Bakelite plate above the MCPGR and the RF shield are not depicted), and c RF circuit modeling of the tunable resonator in ANSYS Electronics Desktop. Coupling capacitors $\left(C_{s}\right)$ are included in the 3D model of the MCPGR.

Fig. 3. Simulated RF characteristics of the tunable resonator. a The RF magnetic field in three perpendicular planes at the center of the MCPGR calculated for $1 \mathrm{~W}$ of applied RF power to the feeding port of the resonator, $\mathbf{b}$ the conversion efficiency of the RF magnetic field and the value of the capacitor $C_{m}$ mimicking varactor diodes for impedance matching as a function of coupling capacitance $C_{s}$, and $\mathbf{c}$ the tunable frequency band as a function of coupling capacitance $C_{s}$. The RF magnetic field was simulated with coaxial lines with a characteristic impedance of $Z_{c}=50 \Omega$. In Figs. 3b and 3c, the black lines and gray lines show the simulated results with 50- and 100-ohm coaxial lines used for the parallel transmission line, respectively.

Fig. 4. Measured RF characteristics of the tunable resonators. a Photographs of the constructed tunable resonator (left: side view, right: top view), b reflection characteristics for the tunable resonator with 50-ohm coaxial lines and coupling capacitance $C_{s}=1.5 \mathrm{pF}$ 
measured at the matching control voltage $V_{M}=6-10 \mathrm{~V}$, and $\mathbf{c}$ the dependence of the resonant frequency on the tuning control voltage $V_{T}$ for tunable resonators with 50-ohm $(\bullet)$ and 100-ohm ( $\mathbf{a})$ coaxial lines.

Fig. 5. EPR spectra of $2 \mathrm{mM}{ }^{15} \mathrm{~N}-\mathrm{PDT}$ measured with the $750-\mathrm{MHz}$ tunable resonator using 100-ohm coaxial lines (top trace) and 50-ohm coaxial lines (bottom trace). The EPR spectra were averaged for 100 scans. The mean values of SNR for three consecutive measurements were 134 and 149 for resonators with 100- and 50-ohm coaxial lines, respectively.

Fig. 6. Three-dimensional EPR imaging of ${ }^{15} \mathrm{~N}-\mathrm{PDT}$ radical. a Photograph of the micro-centrifuge tube containing $2 \mathrm{~mL}$ of $2 \mathrm{mM}^{15} \mathrm{~N}$-PDT solution, b surface-rendered image of the EPR signal, and c slice-selective maps of the EPR signal intensity generated from the 3D image data. EPR imaging was performed with the tunable resonator using 50-ohm coaxial lines for the parallel transmission line. Field-of-view (FOV) of the 3D image is $39 \mathrm{~mm} \times 39$ $\mathrm{mm} \times 39 \mathrm{~mm}$, and the image matrix size is $64 \times 64 \times 64$.

Fig. 7. Three-dimensional EPR imaging of a mouse tumor-bearing hind leg. a Illustration of the mouse tumor-bearing hind leg inside the MCPGR, $\mathbf{b}$ surface-rendered image of the EPR signal in the tumor-bearing leg, and c slice-selective EPR intensity maps. FOV of the 3D image is $33 \mathrm{~mm} \times 33 \mathrm{~mm} \times 33 \mathrm{~mm}$, and the image matrix size is $42 \times 42 \times 42$. 


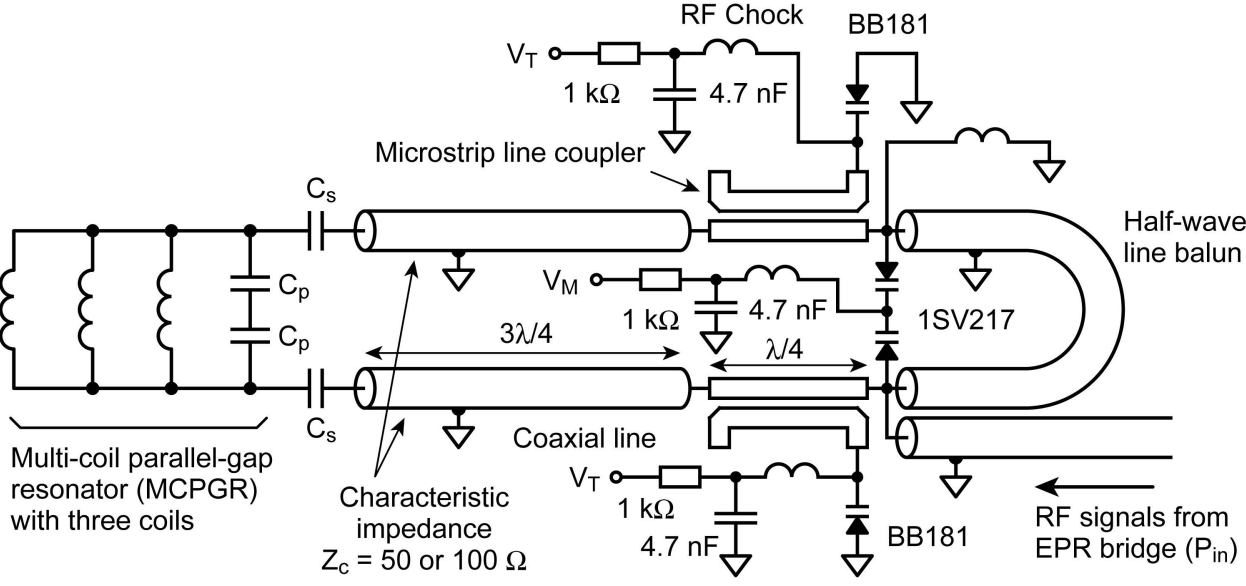




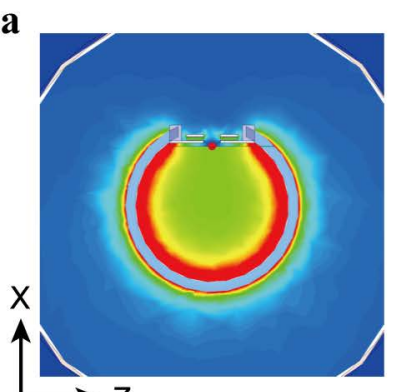

Z

b

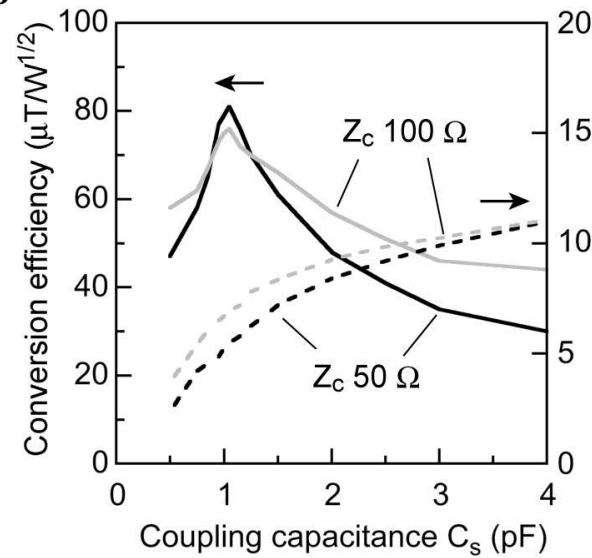

$(A / m)$

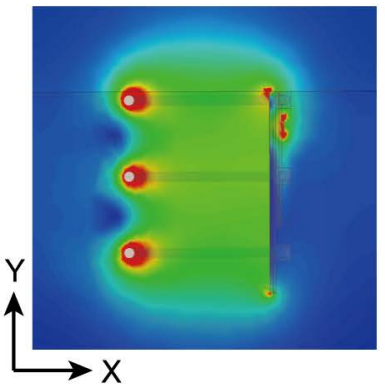

80.0

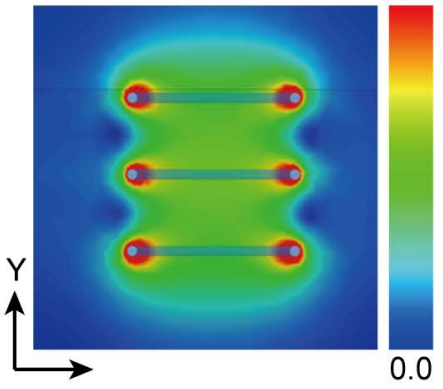

c

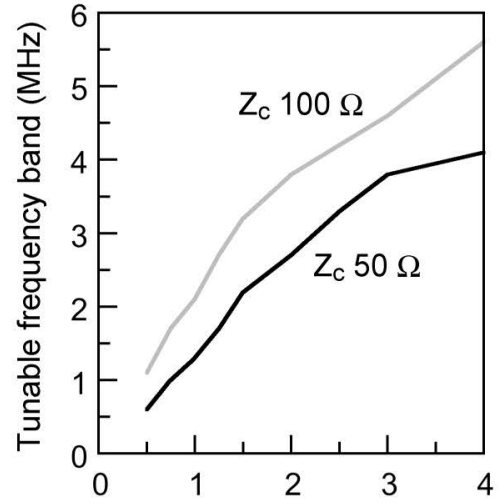

Coupling capacitance $\mathrm{C}_{\mathrm{s}}(\mathrm{pF})$ 
a

Helmholtz coil pair
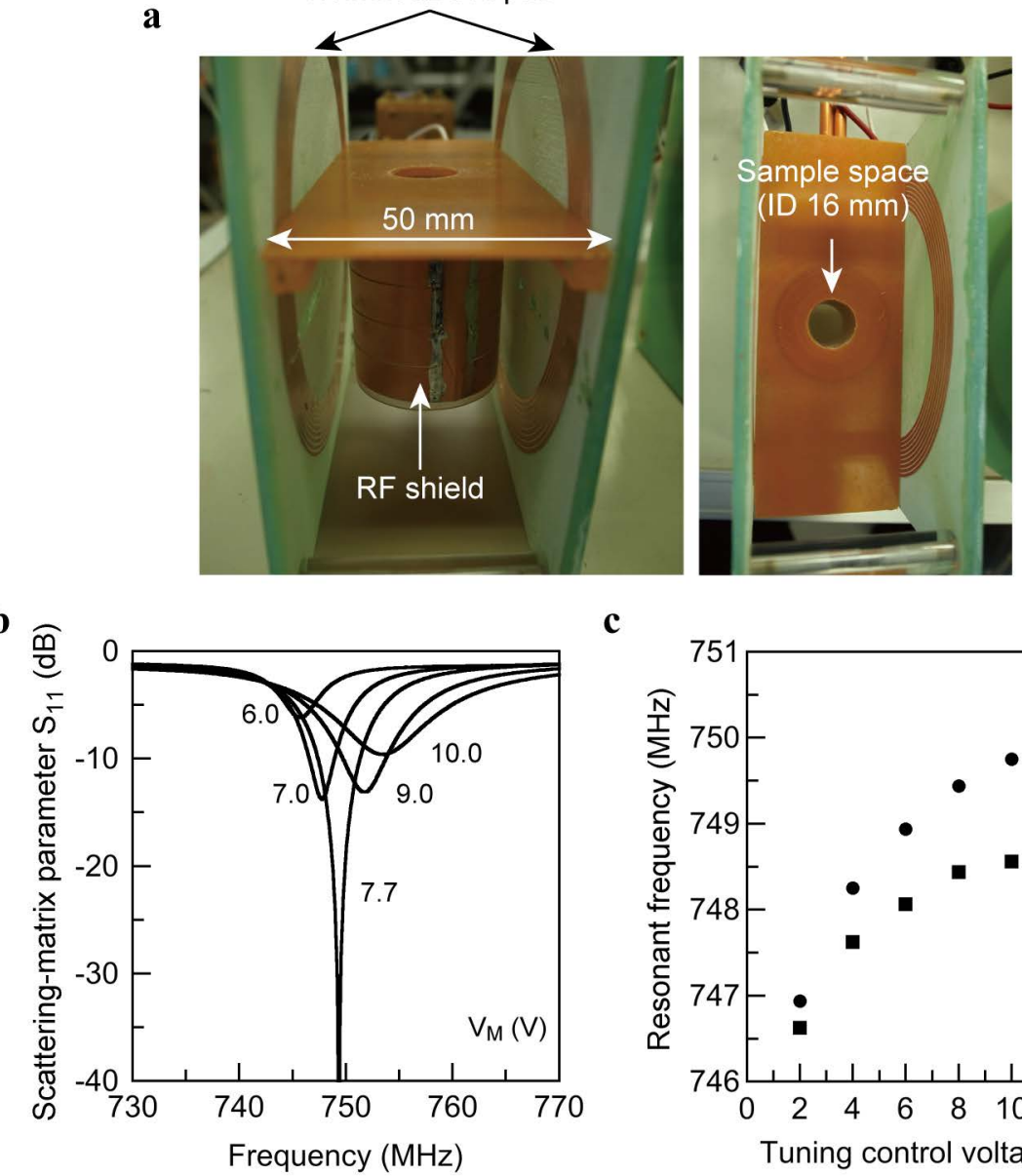

c

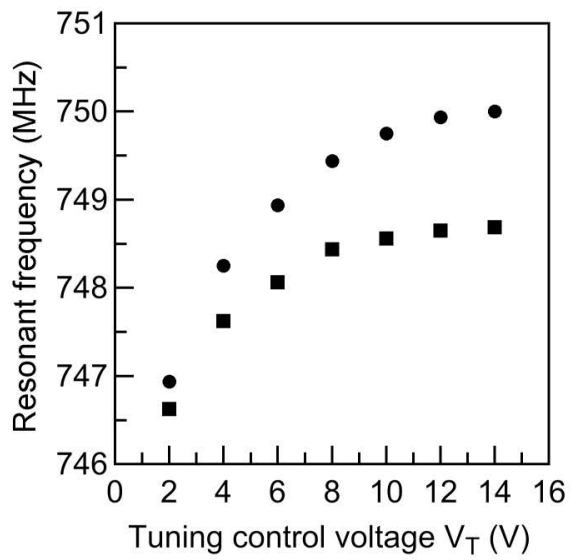




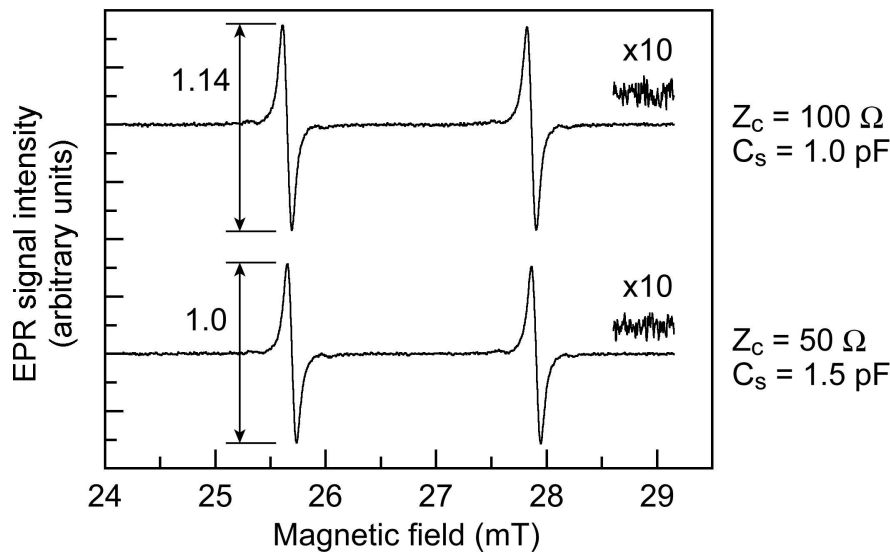



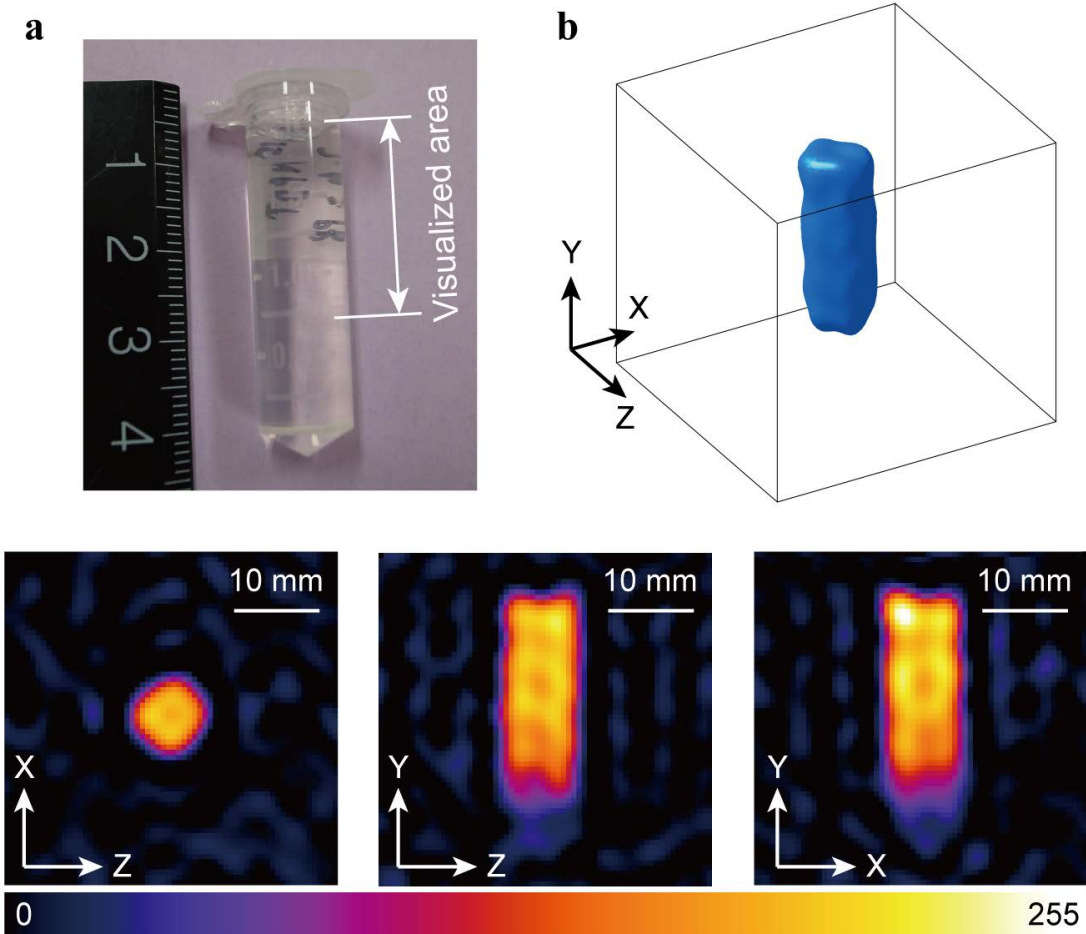

0 

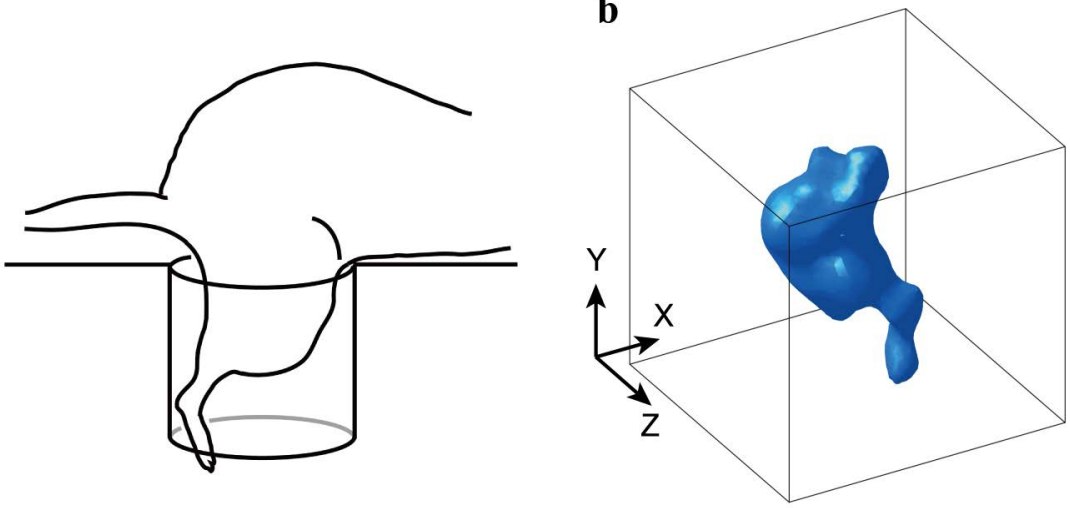

$5 \mathrm{~mm}$

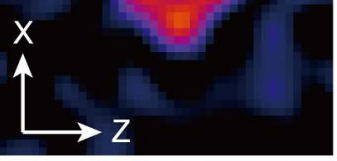

0

\section{$5 \mathrm{~mm}$}

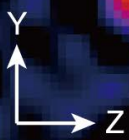

$5 \mathrm{~mm}$

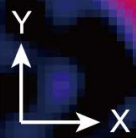

